\title{
High amplitude combustion instabilities in an annular combustor inducing pressure field deformation and flame blow off
}

\author{
Guillaume Vignat; Daniel Durox, Antoine Renaud, Sébastien Candel \\ EM2C Laboratory \\ CentraleSupelec and CNRS \\ Université Paris-Saclay \\ Gif sur Yvette, France \\ Email: guillaume.vignat@centralesupelec.fr
}

This article reports experiments carried out in the laboratory scale annular combustor MICCA-Spray equipped with multiple swirling spray injectors. The experimental setup consists in an air plenum connected to a combustion chamber formed by two concentric cylindrical quartz tubes, allowing full optical access to the flames. A new injection system is introduced and characterized. For a wide range of operating conditions, strong combustion instabilities are observed, but the focus of this article is placed on very high amplitude combustion instabilities coupled by a standing azimuthal mode. New results are obtained using a higher order reconstruction method for the pressure field: its shape is shown to be modified during high amplitude oscillation, leading to asymmetries of the pressure distribution in the system. Flame blow off occurs near the pressure nodal line when a critical level of oscillation is reached. A method is proposed to reconstruct the acoustic velocity field just before blow off occurs and in this way determine the blow off threshold. It is found that the pressure distribution, velocity field and blow off pattern become asymmetric as the amplitude of oscillation increases and that this process is accompanied by a rapid shift in frequency of oscillation. Another notable result is that the heat release rate in the flames on the same side of the nodal line are not perfectly in phase and that the phase differences become larger as the amplitude of oscillation increases.

\section{INTRODUCTION}

New regulations concerning the pollutant emission of aero-engines are pushing manufacturers towards new combustor designs. Burning fuel in a lean, premixed, prevaporized mode has been shown to reduce emissions of harmful nitrogen oxides and soot particles [1]. Swirling injectors are most often adopted to ensure aerodynamic flame stabilization at a distance from boundaries. However, these technological choices lead to a more compact flame, they increase

\footnotetext{
*Address all correspondence to this author.
}

the power density, and reduce the acoustic damping, all factors promoting combustion instabilities.

A large body of research has been dedicated to the investigation, analysis and modeling of the dynamics of swirling flames inside simple combustion systems, and is well summed up in recent reviews [2-4]. The mechanisms involved are fairly complex and interlinked: flow instabilities, equivalence ratio and turbulence fluctuation downstream of an injector will induce fluctuations in the heat release rate of the flame. This unsteady heat release rate is an acoustic source. It will also generate entropy waves. These sources will feed a resonant mode of the system and pressure fluctuation in the chamber will ensue. If one of these modes feeds back perturbations to the flames, it may eventually give rise to oscillations at a frequency that is close to that of the so-called coupling mode. If the process becomes selfsustained one can say that it results from an azimuthal mode coupling when the acoustic mode involved features an azimuthal structure.

Within gas turbine combustors a further degree of complexity is introduced. The combustion chamber in these systems is often annular. The greatest physical dimension of the chamber is then its perimeter, and combustion-acoustic coupling by the first azimuthal acoustic eigenmode of the chamber can occur. These azimuthal combustion instabilities have been observed in the two laboratory-scale annular test rigs investigated in the literature, MICCA at EM2C laboratory [5,6], and the annular rig of the team of Dawson and Worth at Cambridge and later at NTNU $[7,8]$. This kind of coupling was also widely observed in practical gas turbine applications [9-11]. It has been shown that large eddy simulations (LES) could be used to retrieve azimuthally coupled thermoacoustic oscillations in industrial systems [12-14]. These experimental and numerical studies have shown the existence of several degenerate acoustic modes by which coupling can occur: standing, clockwise or counterclockwise spinning, slanted. This behavior is conveniently character- 
ized by the spin ratio $[5,6,15]$, or by plotting the joint probability density function of the amplitude of the clockwise and counterclockwise spinning acoustic components [7,15]. Another characteristic present in all the aforementioned studies featuring swirling injection systems is the intermitent behavior of the resonant mode involving a growth and a decay of the oscillations, as well as switching between spinning and standing modal dynamics.

Compared to longitudinally coupled combustion instabilities, the modeling of such azimuthally coupled systems presents numerous challenges. Reduced order models, either in the form of acoustic network models [5, 16-20] or Helmholtz solvers [21-24], have been used. Both approaches retrieve limit-cycle instabilities observed in the laminar, matrix burner version of the MICCA rig [5, 19-21], but more complex configurations remain a challenge. This modeling work requires an a-priori knowledge of the flame response, usually in the form of a flame describing function (FDF). Its determination may be done by acoustically forcing a flame. In annular burners, especially near the natural frequency of the annulus, this remains a challenge [8]. Usually, a single injector system is used to measure the FDF subject to the assumption that the flames in both systems have the same response. As combustion instabilities are quite sensitive to small changes in flame response as shown analytically in [25] and also with LES [26], a number of parameters, each having a potentially large influence on the flame response, have to be carefully controlled: confinement shape $[27,28]$, wall temperature $[6,29]$, injector geometry $[30,31]$. In a compact flame framework, the response of a flame at a pressure antinode can be modeled using an experimentally determined FDF. This requires measuring the flowrate perturbation of the injector, or alternatively, determining its acoustic impedance. However, flames submitted to transverse velocity perturbation, near the pressure nodal line, have a very different response in the non-linear regime, a topic reviewed by O'Connor et al. [32]. Small variations in flame response, as well as symmetry breaking features also need to be considered, in particular to capture the spinning or standing nature of the mode [16-18, 25,33].

Ghirardo and Juniper [18] show for example that annular systems featuring large transverse velocity fluctuation favor standing acoustic modes by taking into account the effect of these velocity fluctuations in their model. This is coherent with the experiments presented by Prieur et al. [6], where standing acoustic modes appear preferentially when the amplitude reached by the oscillation is high. Additionally, this previous study carried out on the MICCA-Spray configuration indicates that upon reaching a certain value, flame blow off is observed near the pressure nodal line. This behavior is also found by Baillot and Lespinasse $[34,35]$ in a transversally forced geometry.

The present investigation is carried out on the MICCASpray annular combustor [6]. This test rig is an idealized representation of aero-engine combustor configurations. Two major improvements were made to the set-up used in previous study [6]: an array of eight photomultipliers equipped with $\mathrm{OH}^{*}$ narrow-band optical filters gives access to the in- stantaneous heat release rate of one half of the flames in the combustor. Additionally, a new set of injectors, designed after numerous trials, is introduced. A full characterization of this swirled injection system is presented, including measurements of the fuel spray characteristics. The system exhibits a wide range of unstable operating conditions, in terms of thermal power, equivalence ratio and injector bulk velocity, giving rise to many types of thermoacoustic oscillations. Depending on the operating point, either a longitudinal oscillation or an azimuthal oscillation occurs. Limit cycles are observed in the case of the longitudinal oscillation. In other regions of the operating map, azimuthal oscillations take the form of bursts, with lower amplitudes sometimes coupled to spinning modes and with higher amplitudes only under standing mode coupling. The focus is placed in the present article on the analysis of very high amplitude, long bursts coupled by a standing azimuthal mode. The shape of the acoustic field near the backplane of the combustor is investigated using a higher order reconstruction method. Flame blow off during high amplitude standing thermoacoustic oscillations are once again observed. They are studied using high speed chemiluminescence imaging, and dynamic mode decomposition. The instantaneous azimuthal acoustic velocity field is reconstructed and the critical velocity value leading to flame blow off is determined. It is shown that flame blow off induces temperature distribution inhomogeneities that in turn modify the modal structure in the annular combustor. The phase between the instantaneous heat release rate in each flame and the acoustic pressure is examined and it is shown that this quantity changes from flame to flame on the same side of the nodal line and that these variations in phase become larger as the amplitude of oscillation increases.

\section{EXPERIMENTAL SETUP}

In this section, we successively describe the MICCASpray test rig, the injection units and the resulting velocity distributions and the diagnostic tools.

\section{MICCA-Spray Test Rig}

The MICCA-Spray test rig (Fig. 1) comprises an annular combustor with spray and swirled injectors meant to be representative of the combustor of a small helicopter engine. However, this device operates at atmospheric pressure. Previous studies on combustion instabilities in this rig include [6] in the spray flame configuration, and [5,36,37] in a previous propane-air configuration. The rig comprises an annular plenum, fed with air at ambient temperature by eight evenly distributed channels, and closed on the top and the bottom by water-cooled stainless steel plates. The plenum is $80 \mathrm{~mm}$ high, and has an inner diameter of $280 \mathrm{~mm}$ and an outer diameter of $420 \mathrm{~mm}$. Sixteen injectors are mounted on the upper plate of the plenum, which also forms the backplane of the combustion chamber. The combustor itself is an open-ended annulus with two concentric cylindrical vertical walls. The inner vertical boundary of the combustor is a quartz tube, $8 \mathrm{~mm}$ thick, $200 \mathrm{~mm}$ high, with an outer di- 

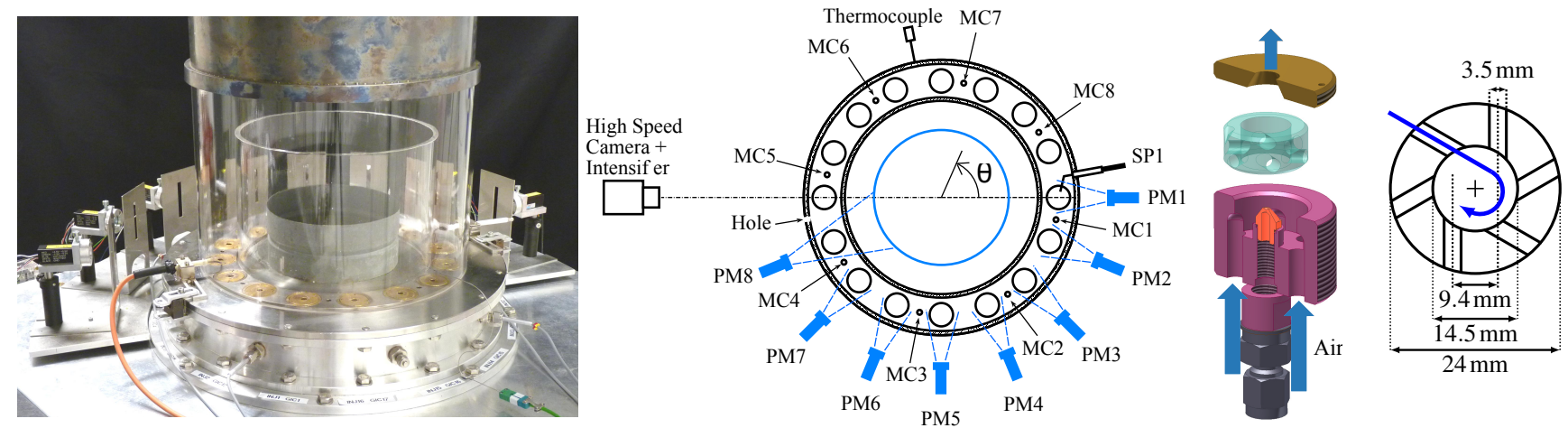

Fig. 1: From left to right: (1) Photography of the MICCA-Spray test rig. (2) Schematic view of the chamber backplane showing the locations of the camera, of the thermocouple, of the different microphones MCx in the chamber and MPx in the air plenum, of the phtomultipliers PMx and of the spark plug SP1. A hole is drilled in the quartz wall opposite to spark plug SP1. Dashed blue lines show the field of view of the photomultipliers. A steel tube is added in the center to block the luminosity of the opposite flames when using the photomultipliers. This tube is taken away when the camera is in use. The reference point for the azimuthal coordinate $\theta$ in the chamber is shown as the center of injector 1 , which is initially ignited by spark plug SP1. $\theta$ is chosen positive in the counterclockwise direction. (3) Exploded view of the injector and its components. In purple, the main body, in orange the liquid fuel atomizer, in translucide teal the tangential air swirler and in gold the injector outlet, which is flush with the chamber backplane. (4) Schematic view of the swirler seen from above indicating the main dimensions of this component.

ameter of $300 \mathrm{~mm}$. The outer wall, $700 \mathrm{~mm}$ high and with an inner diameter of $400 \mathrm{~mm}$, is made in two parts: the bottom is a $300 \mathrm{~mm}$ long quartz tube, $6 \mathrm{~mm}$ thick; the upper is a $400 \mathrm{~mm}$ long heat resistant steel tube. The air flow to the experiment is metered using two Bronkhorst EL-Flow mass flow controller, leading to a relative accuracy better than $1 \%$. The fuel flow is measured by a Bronkhorst CORI-Flow controller, with a relative accuracy of $0.5 \%$. $9 \mathrm{~mm}$ holes are drilled in the outer quartz tube, $15 \mathrm{~mm}$ above the back plane, to accommodate spark plugs (see Fig. 1). For the experiment presented in this paper, spark plug SP1 is plugged in and used for ignition, while the opposite hole is left empty. The tip of the spark plug is placed approximately $15 \mathrm{~mm}$ from the center of an injector.

\section{Injectors}

The spray swirl injection system used in this study is a modified version of the injector used by Prieur and al. [6]. It is designed to obtain a higher swirl number of $S=0.71$. This is assumed to favor stronger self-sustained combustion instabilities on a wider range of operating conditions. In the exploded view of the injection system shown in Fig. 1 the air of the plenum flows from the bottom, passes through six axial channels $6 \mathrm{~mm}$ in diameter, and then through the six tangential channels of the swirler, depicted schematically with dimensions in the rightmost part of Fig. 1. The air is set in a rotational motion at this stage, and exits to the combustor through a small conical section, with an $8 \mathrm{~mm}$ diameter at the outlet of the injector. Liquid $n$-heptane is injected by an atomizer set $7 \mathrm{~mm}$ in recess with respect to the dump plane. A hollow cone spray is formed by this device.

The experimental characterization of the injector has been performed on a single injector rig, at an operating point corresponding to $\left(\mathcal{P}=111 \mathrm{~kW}, \phi=0.85, u_{b}=42.7 \mathrm{~m} \mathrm{~s}^{-1}\right)$ in MICCA-Spray, where the bulk velocity through an injector is defined as the 1D averaged velocity at the injector's outlet under cold flow conditions $u_{b}=\dot{m} /\left(\pi \rho R_{i n j}^{2}\right)$.

The swirl number of these injectors is deduced from velocity profiles measured $2.5 \mathrm{~mm}$ above the dump plane in the absence of any confinement, under non-reactive conditions, and in the absence of fuel. The air is seeded with oil droplets approximately $2 \mu \mathrm{m}$ in diameter, and the velocities are measured using a two-component Dantec Dynamics FlowExplorer Phase Doppler Anemometer. The theoretical optical probe volume of this system measures $0.14 \mathrm{~mm} \times 0.14 \mathrm{~mm} \times 0.23 \mathrm{~mm}$. Measurements are spaced every $0.25 \mathrm{~mm}$. Uncertainty on the measured mean velocities is less than $0.8 \mathrm{~ms}^{-1}$. The mean velocity profiles for this injection system are shown in Fig. 2. The experimental swirl number $S=0.71$ is calculated using the conventional expression [38]:

$$
S=\frac{\int_{0}^{2 R_{i n j}} \overline{U_{\theta}} \overline{U_{x}} r^{2} d r}{R_{i n j} \int_{0}^{2 R_{i n j}}{\overline{U_{x}}}^{2} r d r}
$$

Droplet size measurements are also performed on the fuel spray, $2.5 \mathrm{~mm}$ above the dump plane in the absence of any confinement, and under non-reactive conditions. The Sauter mean particle diameter is $d_{32}=18.0 \mu \mathrm{m}$, while the mean particule diameter is $d_{10}=4.5 \mu \mathrm{m}$. The head loss of the injection unit was also characterized under non-reactive conditions, using a Kimo MP111 differential pressure gauge, with a $1 \%$ relative precision. The pressure loss across the injector follows the standard scaling law $\Delta p=1 / 2 \rho \sigma u_{b}^{2}$, with 


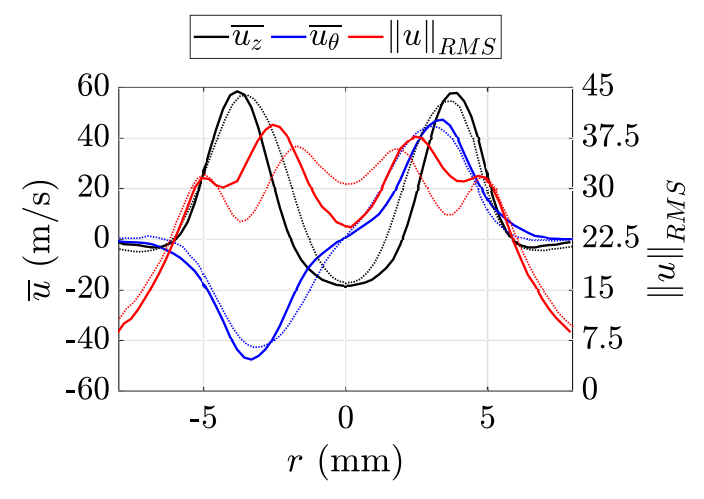

Fig. 2: Left axis: mean velocity profiles measured $2.5 \mathrm{~mm}$ downstream of the injector outlet using LDV. The axial component is shown in black, the tangential component in blue. Right axis: overall turbulent velocity. The profiles for the injector used in the present study are shown in solid lines, those for the injector used in [6] in dotted lines. The injector used in this study features a swirl number $S=0.71$, the one used in [6] $S=0.68$. These swirl numbers were determined from the profiles that are presented here.

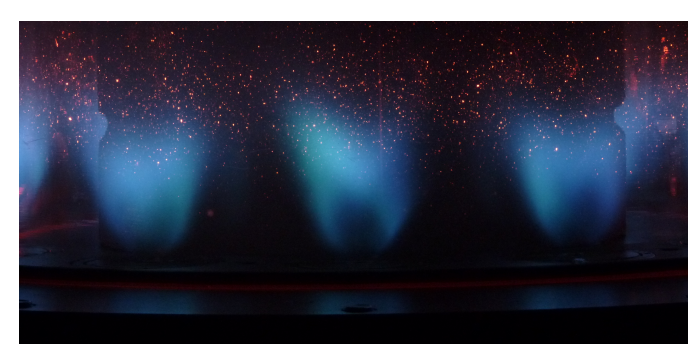

Fig. 3: Direct chemiluminescence image of several stable flames in MICCA-Spray at operating point $(\mathcal{P}=$ $\left.107 \mathrm{~kW}, \phi=1.04, u_{b}=33.4 \mathrm{~m} \mathrm{~s}^{-1}\right)$. The exposure time is $100 \mathrm{~ms}$ so that the images are time-averaged. The flames have a typical "M"-shape. Slight differences can be observed from one flame to the other. The system is not perfectly rotationally symmetric due to the presence of the spark plugs, and differences in fuel atomization between each injector.

$\sigma=5.1$ being the head loss coefficient. Figure 3 shows chemiluminescence images of stable flames in the combustion chamber. The upper part of the outer boundary of the combustor (steel prolongating tube) has been removed to suppress the thermoacoustic oscillation.

\section{Diagnostic Tools}

In addition to the diagnostic tools found on the single injector rig, and presented in the previous section, the MICCASpray experimental rig is equipped with several sensors for the analysis of self-sustained thermoacoustic oscillations. The sensor positions are indicated in Fig. 1.

A total of 12 Bruel \& Kjaer type 4938 microphones with type 2670 preamplifiers are used to measure acoustic pres- sure signals. Their relative accuracy is $1 \%$ and their cutoff frequencies are $70 \mathrm{kHz}$ and $15 \mathrm{~Hz}$. All signals are sampled at $32768 \mathrm{~Hz}$. Four of these microphones, designated as MP1 to MP4, are plugged onto the plenum, and eight of these sensors, designated as MC1 to MC8, are mounted on waveguides. These are connected to the backplane of the combustion chamber with their orifices set at equal distance of two injectors. The waveguides are terminated by a $25 \mathrm{~m}$ long flexible tube to avoid acoustic reflexions from the downstream end of the device. The microphones are located at $170 \mathrm{~mm}$ from the dump plane of the combustor. This arrangement induces a time lag $\tau_{m-b}=0.46 \mathrm{~ms}$ that can be estimated by taking into account the temperature prevailing inside the plenum, which was measured at $55^{\circ} \mathrm{C}$. This delay is taken into account when synchronizing the signals recorded during experiments. The signals delivered by these microphones are used to reconstruct the acoustic pressure field at each azimuthal position within the chamber and plenum. An array of eight photomultipliers also equips the experimental rig. They are positioned outside the annulus. Each photomultiplier is pointing at an individual flame, and is equipped with a mask acting as a spatial filter. A steel tube, $90 \mathrm{~mm}$ high and $205 \mathrm{~mm}$ in diameter, is placed within the inner quartz tube of the chamber (see the photography in Fig. 1). This tube and these masks ensure that each photomultiplier only detects light emitted by a single flame. Each photomultiplier is equipped with an optical filter centered on the chemiluminescence band of the $\mathrm{OH}^{*}$ radical. These are pass-band filters centered on $308 \mathrm{~nm}$ with a bandwidth at half maximum of $12 \mathrm{~nm}$. The light emission signal measured by these instruments can be used to roughly estimate the instantaneous heat release rate of each flame. It is worth noting that, even under these non-premixed conditions, one may assume that the PM signal intensity provides to some extent an indication on the combustion intensity, which is linked to the flame luminosity. This was shown experimentally using a quasisteady approach by Mirat et al. [39] and successfully applied in [40]. A K-type thermocouple is also present on the outer wall of the combustor to measure the local temperature $T_{\text {wall }}$, in-between two injectors, $36 \mathrm{~mm}$ above the backplane.

The global motion of the flame is captured using a high speed intensified camera, without any optical filter. The camera, a Photron FastCam APX-i2, uses a CMOS sensor with a $512 \times 256$ pixels resolution, and an 8-bit gray level resolution. Recordings are performed at 6000 frames per second with an exposure time of $166 \mu$ s. The camera is positioned as shown in Fig. 1 in order to have a bird's eye view of the whole rig. Its axis is aligned with the position of the spark plugs, which are close to the nodal line during oscillations coupled by a standing azimuthal mode.

\section{THERMOACOUSTIC OSCILLATIONS COUPLED BY A STANDING AZIMUTHAL MODE}

Thermoacoustic oscillations coupled by a standing azimuthal acoustic mode are observed in the MICCA-Spray configuration for numerous operating conditions. The corresponding intermittent oscillations take the form of long 

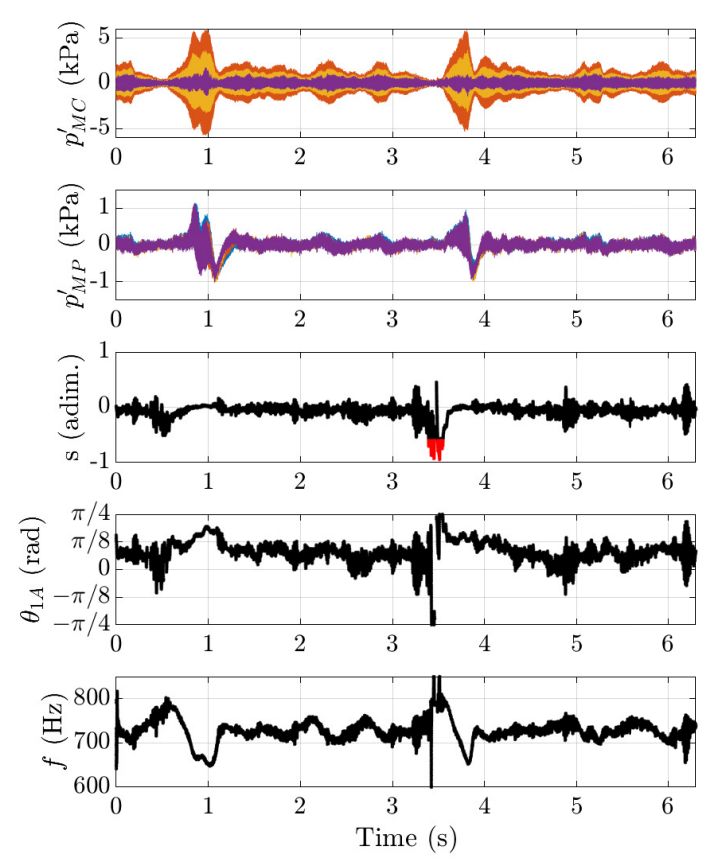

Fig. 4: Typical time series of thermoacoustic oscillations in the MICCA-Spray rig for operating conditions $\left(\mathcal{P}=128 \mathrm{~kW}, \phi=1.11, T_{\text {wall }}=828 \mathrm{~K}\right)$. On top, the raw acoustic pressure signals in the chamber $p_{M C}^{\prime}$ are shown for microphones MC1-MC4. Then the raw acoustic pressure signals in the plenum $p_{M P}^{\prime}$, followed by the time evolution of the spin ratio $s$, calculated according to Eq. (2). The position of the pressure nodal line of the first in space azimuthal acoustic mode $\theta_{1 A}$ is shown using the angle $\theta$ defined in Fig. 1. The instantaneous frequency $f$ of the pressure signal is presented at the bottom. The colors for microphones MCx and MPx are shown in Fig. 5.

bursts several hundred millisecond long. The present article focuses on this mode of oscillation which features relatively low growth rates allowing time resolved processing of the microphone and photomultiplier signals. A typical time series recording is presented in Fig. 4, where pressure signals in the chamber (dubbed MCx) are shown at the top while pressure signals in the plenum (MPx) appear directly underneath. The bottom part of this figure displays the instantaneous frequency of the pressure signals in the chamber. The instantaneous frequency is computed from the pressure signal detected by the chamber microphone $\mathrm{MC} 1$, filtered using a bandpass 8th order Butterworth filter, with cut-off frequencies $600 \mathrm{~Hz}$ and $950 \mathrm{~Hz}$. The analytical signal is then deduced with a Hilbert transform. The frequency is obtained by differentiating the phase of this analytical signal. This method compares quite favorably with time resolved spectrograms obtained using short-time Fourier transforms (not shown here). These spectrograms feature a peak that is shifting in time from 800 to $620 \mathrm{~Hz}$. This peak is dominant by at least $20 \mathrm{~dB}$ over the other components in the spectrum.
Figure 5 shows three representative, zoomed in sequences. Sequences (a) and (b) are extracted from the longer sequence displayed in Fig. 4, while sequence (c) originates from a separate experiment during which the high speed camera was used.

As already observed by Prieur et al. [6], the instantaneous frequency is consistently found to decrease during the growth of a thermoacoustic oscillation, and increase as the amplitude of the thermoacoustic oscillation decreases. The spin ratio presented in Fig. 5 is that of the first azimuthal component, and is calculated using the definition and method derived by Bourgouin et al. [41]

$$
s=\frac{\left\|a_{+1}\right\|-\left\|a_{-1}\right\|}{\left\|a_{+1}\right\|+\left\|a_{-1}\right\|}
$$

where $a_{+1}$ and $a_{-1}$ respectively designate the amplitudes of the counterclockwise and clockwise spinning components of the first azimuthal acoustic mode (defined in Eq. (4)). All three bursts are coupled by a standing mode. Figure 5 also displays the position of the pressure nodal line of the first azimuthal acoustic mode. It is found to move slightly during a thermoacoustic burst, by approximately $\pi / 8 \mathrm{rad}$, which corresponds to the angle between two injectors. The nodal line is nearly aligned with the diameter on which the spark plug and the opposing hole are found (see Fig. 1). This behavior is also found when two opposing spark plugs are present in the combustor. This characteristic is used in practice to freeze the position of the nodal line, and adequately position the high speed camera.

\section{SPATIAL STRUCTURE OF THE ACOUSTIC PRES- SURE FIELD}

Using the eight bandpass-filtered $(600 \mathrm{~Hz}<f<950 \mathrm{~Hz})$ pressure signals MC1-MC8, the spatial structure of the acoustic pressure field in the chamber can be reconstructed in the form of a Fourier series. For the present work, this reconstruction is carried out up to order three in azimuthal angle. In this framework, the analytical signal of the acoustic pressure field $p^{\prime}(\theta, t)$ near the backplane of the combustor is represented as a finite Fourier series

$$
p^{\prime}(\theta, t)=\sum_{m=-M}^{M} \exp (i m \theta) \eta_{m}(t)
$$

where $M$ is the number of Fourier components (in the present application, $M=3$ ), $\theta$ is the azimuthal coordinate defined in Fig. 1, and taken positive in the counterclockwise direction. $\eta_{m}(t)$ is the projection of the analytical signal $p^{\prime}(\theta, t)$ (determined by means of an Hilbert transform) on the Fourier basis. In practice, in the case of combustion instabilities examined in this paper, the power spectral density of the pressure signals (not shown here) presents a strong peak corresponding to the first azimuthal and longitudinal, $1 \mathrm{~A} 1 \mathrm{~L}$ acoustic 

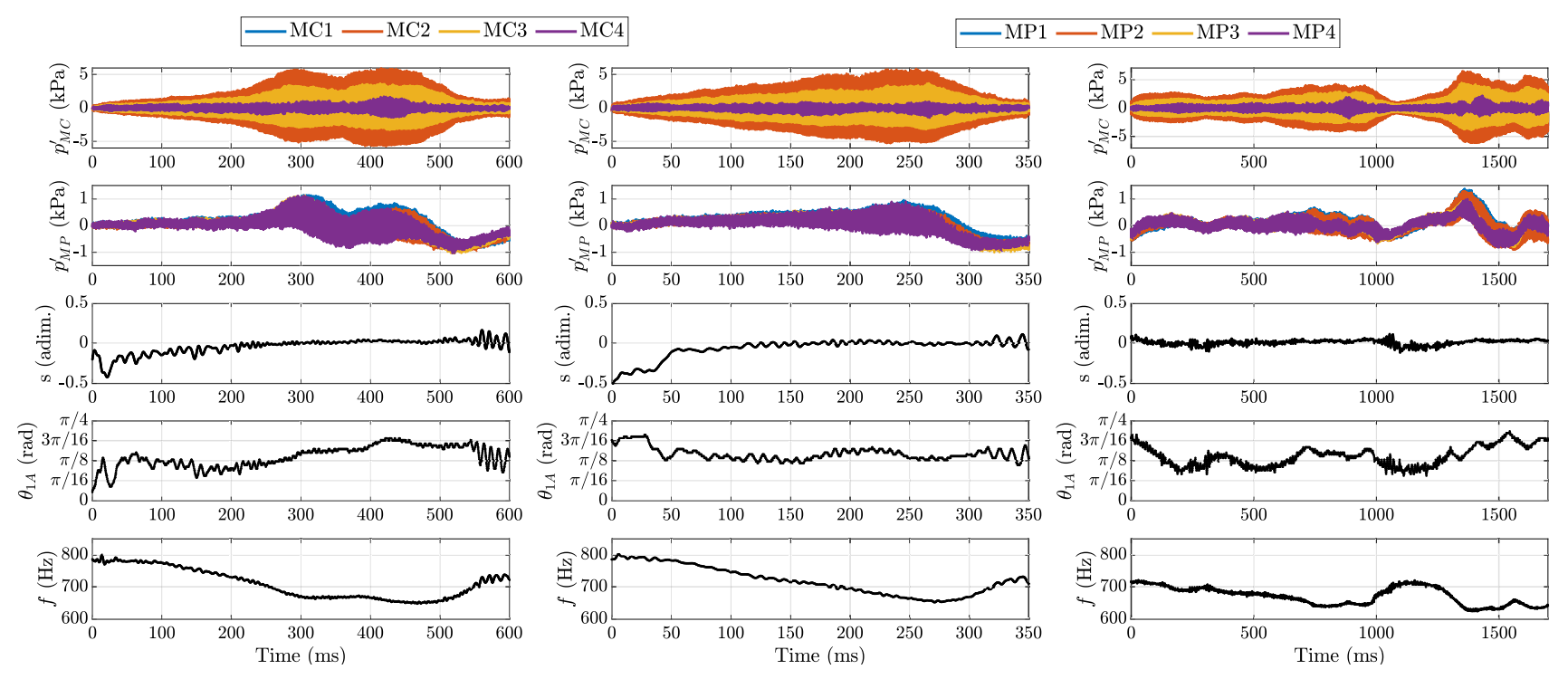

(a) $\left(\mathcal{P}=128 \mathrm{~kW}, \phi=1.11, T_{\text {wall }}=828 \mathrm{~K}\right)$
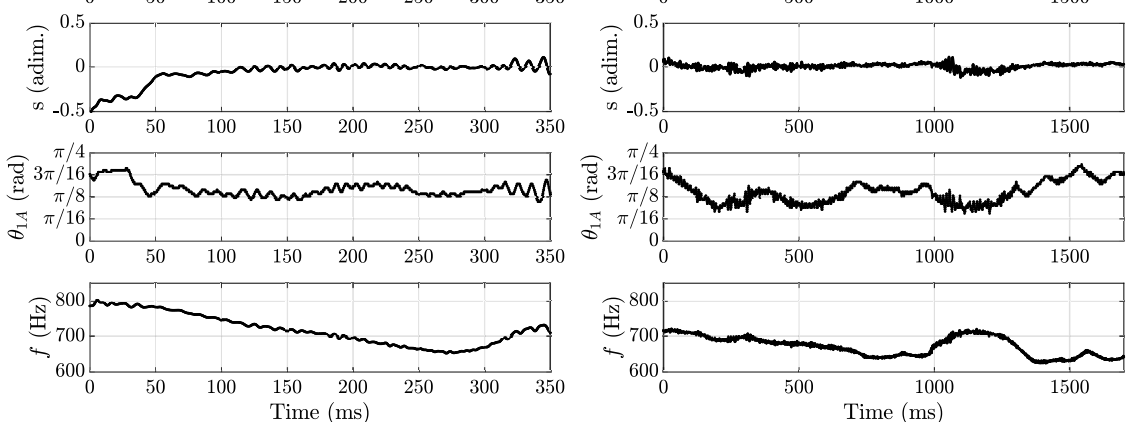

(b) $\left(\mathscr{P}=128 \mathrm{~kW}, \phi=1.11, T_{\text {wall }}=828 \mathrm{~K}\right)$

(c) $\left(\mathcal{P}=117 \mathrm{~kW}, \phi=1.09, T_{\text {wall }}=811 \mathrm{~K}\right)$

Fig. 5: Three thermoacoustic oscillation bursts coupled by a standing acoustic wave. The operating condition is indicated underneath each sequence. Note that sequences (a) and (b) are two distinct time sequences obtained at the same operating conditions, extracted from the longer time series in Fig. 4. For sequences (a) and (b), the photomultiplier array was used to acquire flame chemiluminescence data, while for sequence (c), the high speed camera was used.

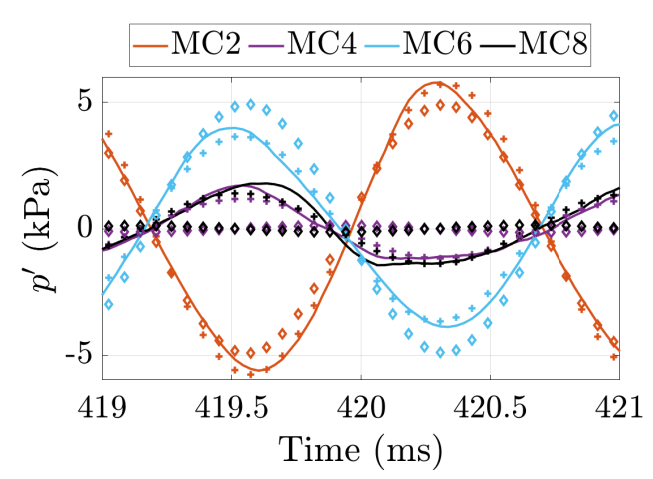

Fig. 6: Pressure signals from Seq. (a) of Fig. 5. At this instant, the thermoacoustic oscillation executes a quasi limit cycle, and the nodal line of the first azimuthal mode is nearly aligned with microphones MC4 and MC8. The measured pressure signals are shown in plain lines, the signals reconstructed using the first order in space method of $[6,41]$ appear as diamond symbols, the signals reconstructed using the third order in space method used in the present article are plotted as cross symbols.

mode. This peaks dominates by at least $20 \mathrm{~dB}$ over other frequencies, so that Eq. (3) can be simplified into

$$
p^{\prime}(\theta, t)=\sum_{m=-3}^{3} a_{m}(t) \exp (i[m \theta-2 \pi f(t) t])
$$

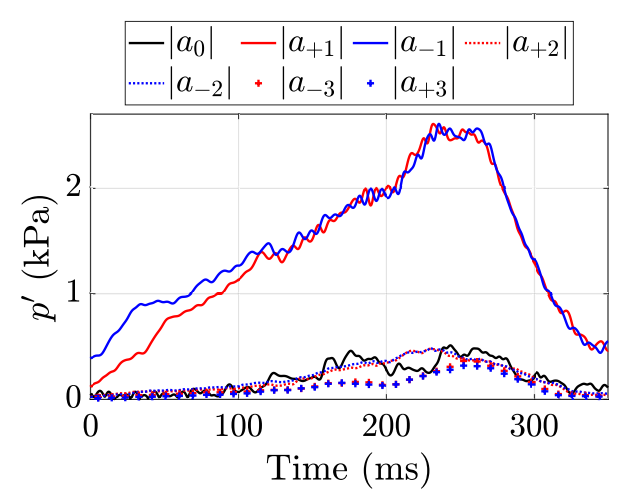

Fig. 7: Seq. (b) of Fig. 5. Time evolution of the amplitude of the seven reconstructed acoustic components defined in Eq. (3).

Complex amplitudes $a_{m}(t)$ are defined for each $\mathrm{m}^{\text {th }}$ order spatial component. $f(t)$ is the instantaneous frequency of the signal. The least square method used in previous works $[6,41]$ is adapted for this higher order reconstruction. This higher order approach notably improves the fidelity of the pressure field reconstruction during high amplitude oscillations. For the thermoacoustic instabilities presented in Fig. 5, the time-averaged Pearson product-moment correlation coefficient between the measured and reconstructed pressure signals is $R_{1 A}^{2}=97.3 \%$ using the first order reconstruction method, and $R_{3 A}^{2}=99.7 \%$ using the third order spatial reconstruction introduced in the present article. Although the value of $R_{1 A}^{2}$ might appear perfectly acceptable at first, Fig. 6 


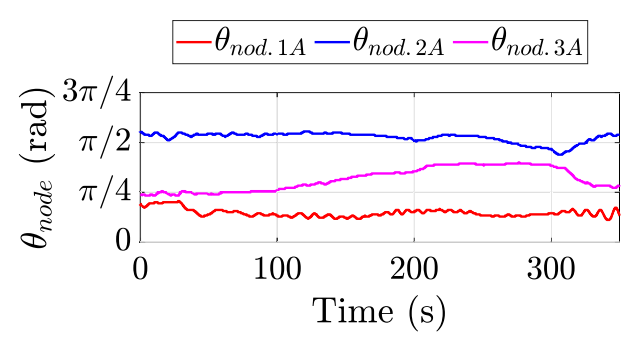

Fig. 8: Seq. (b) of Fig. 5. Time evolution of the position of the nodal line associated with each standing component. For the $m^{\text {th }}$ order component, $m$ nodal line co-exist. $\theta_{\text {node }}$ refers to the angular position of the pressure node in the interval $[0 ; \pi / 2 m[\mathrm{rad}$.

shows that there are reconstruction errors when using a first order reconstruction method (diamonds in Fig. 6). This is particularly true in the vicinity of the nodal line, close to microphones MC4 and MC8 in the present case. The third order method (crosses in Fig. 6) greatly improves the reconstruction of the spatial structure of the pressure mode. Additionnaly, the root mean square acoustic pressure in the annulus, defined as the spatially averaged RMS value of the pressure, is easily computed using Eq. 5.

$$
p_{R M S}^{\prime}(t)=\frac{1}{\sqrt{2}}\left[\sum_{m=-M}^{M} a_{m}^{2}(t)\right]^{1 / 2}
$$

The time evolution of the amplitude of the reconstructed waves is shown in Fig. 7. For all three orders, the amplitude of the clockwise and counterclockwise spinning components are nearly equal, indicating that a mostly standing acoustic wave with spin ratio close to zero is found. During very high pressure oscillations $\left(p_{R M S}^{\prime}>4 \mathrm{kPa}\right.$ ), the sum of the peak amplitudes of the second and third order components is found to be of the order of 20 to $30 \%$ of that of the first order wave. All acoustic components have the same instantaneous frequency, that of the first order wave, shown at the bottom of Fig. 5.

Figure 8 shows the position of the nodal line for each standing component. These lines are staggered. In Fig. 6, microphones MC4 and MC8 are found to be very close to the nodal line of the first order azimuthal component. Yet, pressure fluctuations at their location reach an amplitude of $1.7 \mathrm{kPa}$. This is a consequence of the staggered nature of the nodal lines: flames at the pressure nodal line of the first component will be submitted to transverse velocity fluctuations from this first component, but also to pressure fluctuations from the higher order components; flames at the pressure antinode of the first component will be submitted to pressure fluctuations from this first component, but also to transverse velocity fluctuations from the higher order components.

\section{FLAME blow off DURING LARGE AMPLITUDE THERMOACOUSTIC OSCILLATIONS}

In a previous work [6], it was found that large amplitude thermoacoustic oscillations coupled by a standing acoustic mode could destabilize flames formed by injectors located near the pressure nodal line and that this could lead to partial blow off. The pressure nodal line corresponds to an antinode of the acoustic azimuthal velocity component inducing a strong transverse motion of the flame and its root. A theoretical framework was also proposed to evaluate the blow off limit. Blow off of flames located near the pressure nodal line was also found to occur in the new configuration of the MICCA-Spray combustor investigated in the present article, under oscillations coupled by a standing acoustic mode. Thus, this analysis is continued here by first examining high speed chemiluminescence images, by next estimating the critical velocity fluctuation threshold corresponding to blow off, and finally by considering the consequences of blow off in terms of modal distortion.

\section{High Speed Imaging}

In this section, sequence (c) from Fig. 5 will be analyzed in some details using high speed chemiluminescence imaging. In Fig. 9, three short sequences, each $100 \mathrm{~ms}$ long, are isolated. They feature a stable, quasi limit-cycle oscillation at three different amplitudes. Average chemiluminescence images are shown in the top of Fig. 9. In Fig. 9(b), none of the flames are blown-off, but flame 1 appears to be weaker compared to the others. The amplitude of the pressure oscillation is much higher in Fig. 9(c), and flames 1, 6, and 7 are blown-off. Flames 8 and 16 are nearly blown-off as well. The highest amplitude is reached in Fig. 9(d), where a total of five flames are blown-off: 1, 6, 7, 8, and 16. An asymmetry in the flame behavior can already be observed.

In order to go further in the analysis of these images, it is convenient to use the dynamic mode decomposition (DMD) introduced by Schmid [42]. A DMD mode is written as:

$$
\mathcal{M}(\boldsymbol{x}, t)=\mathcal{K}(\boldsymbol{x}) \mathrm{e}^{i \varphi(\boldsymbol{x})} \mathrm{e}^{(a+2 i \pi f) t}
$$

where $\mathcal{K}$ is the local amplitude of the mode at position $x, \varphi$ its local phase, $a$ its growth rate and $f$ its temporal frequency. All DMDs are obtained by processing 600 images, corresponding to $0.1 \mathrm{~s}$. The most energetic mode, corresponding to the thermoacoustic oscillation at the frequency of the standing acoustic wave, is shown in the second row of Fig. 9. The spatial part of the mode is displayed with both the amplitude $\mathcal{K}(\boldsymbol{x})$ and phase $\varphi(\boldsymbol{x})$ using the colormap in Fig. 9(e). The color indicates the phase of the mode, the light intensity its amplitude.

\section{Estimation of Critical Velocity Threshold}

Blow off is first observed for peak acoustic pressure fluctuations reaching $2 \mathrm{kPa}$ to $2.5 \mathrm{kPa}$ in the combustor. As this phenomenon occurs near the pressure nodal line, which is an 


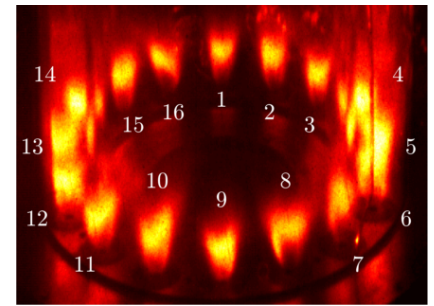

(a) Stable combustor for reference

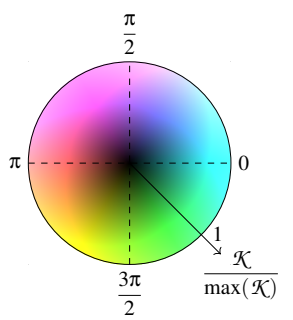

(e) DMD colormap (see Eq. (6))

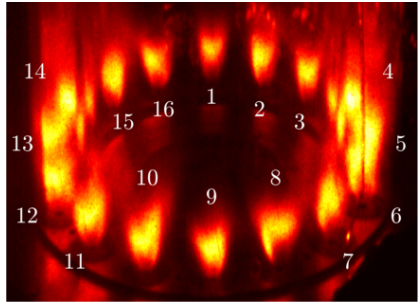

(b) $t=1.05 \mathrm{~s}$ to $t=1.15 \mathrm{~s}$ $p_{\max }^{\prime} \approx 1.0 \mathrm{kPa}$

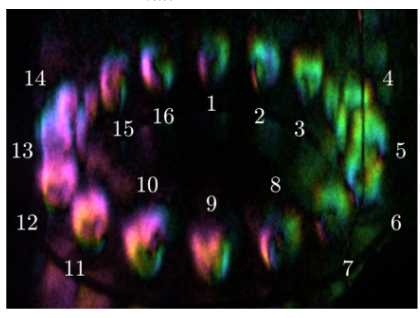

(f) $f=712 \mathrm{~Hz}$

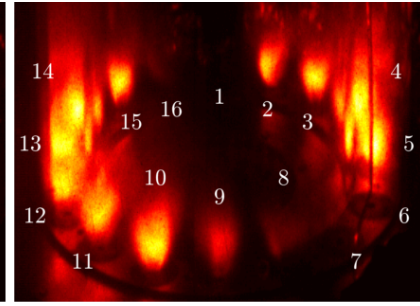

(c) $t=0.7 \mathrm{~s}$ to $t=0.8 \mathrm{~s}$ $p_{\text {max }}^{\prime} \approx 3.8 \mathrm{kPa}$

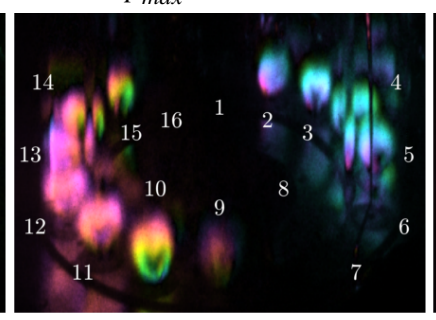

(g) $f=642 \mathrm{~Hz}$

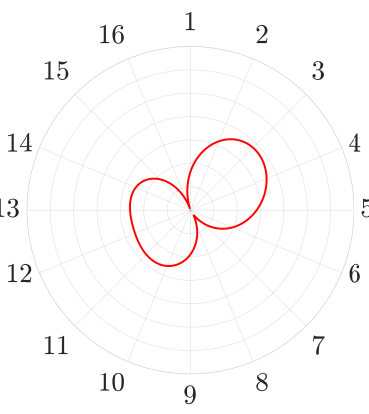

(j)

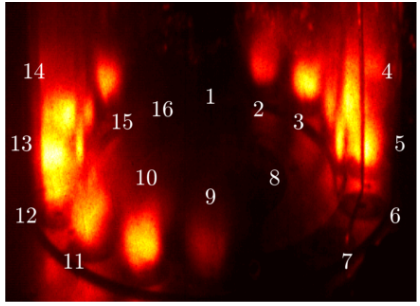

(d) $t=1.35 \mathrm{~s}$ to $t=1.45 \mathrm{~s}$ $p_{\text {max }}^{\prime} \approx 6.1 \mathrm{kPa}$

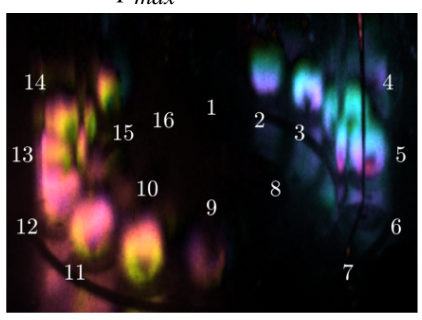

(h) $f=631 \mathrm{~Hz}$

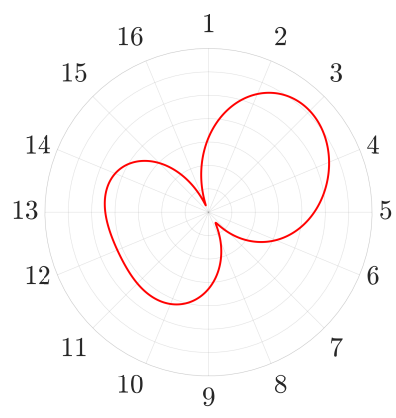

(k)

Fig. 9: Data is extracted from sequence (c) of Fig. 5. 9(a) Time averaged chemiluminescence image of the combustor in the absence of thermoacoustic oscillations. The flames are numbered from 1 to 16 , flame 1 corresponding to the flame near the spark plug in Fig. 1. 9(b)-9(d) Time averaged chemiluminescence image of the combustor. Three $100 \mathrm{~ms}$ long sequences are isolated. These correspond to carefully chosen periods during which the oscillation is constant in amplitude and frequency. 9(f)-9(h) Spatial representation in amplitude and phase of the most energetic DMD mode of each image sequence. The phase reference is set by the pressure signal from microphone MC2 located between flames 2 and 3. 9(i)-9(k) Spatial structure of the acoustic pressure $\hat{p}$. It is reconstructed using the method described in Eq. (3). The numbers in azimuth correspond to the injectors seen in the images. The amplitude is shown by the radial position of the curve, each circle represents $1 \mathrm{kPa}$.

acoustic velocity antinode, it is particularly relevant to determine the azimuthal acoustic velocity. A method to do so from pressure measurements is described in Appendix A. As this method requires the density field to be known, the analysis is limited to the instants just before the blow off of the first flame, as a uniform temperature field hypothesis can then be made.

Figure 10 shows the acoustic pressure and velocity fields computed at $t=106 \mathrm{~ms}$ for sequence (c) from Fig. 5. This is just after blow off of flame 1 a situation identified in the high speed images. All other flames are still attached, so that the acoustic velocity can be retrieved using a uniform temperature model. The acoustic pressure appears to be asymmetric between the two sides of the nodal line, and the acoustic velocity is even more asymmetric, being $35 \%$ higher near injector 1 than near injectors 8 or 9 . These ve- locity fields asymmetries are also found during other similar blow off events.

The critical blow off velocity $\hat{v}_{\theta}^{b o}$ at which flame 1 will be blown-off can be determined from Fig. 10. From this figure, the blow off velocity of flame 1 is estimated at $\hat{v}_{\theta}^{b o} \approx 16 \mathrm{~ms}^{-1}$ for $T=1550 \mathrm{~K}$. $T$ is not well know. $T=1550 \mathrm{~K}$ is estimated from the frequency of the thermoacoustic oscillation. However, one may reasonably consider that $T$ lies within the range $1500-1800 \mathrm{~K}$, leading to $15.5 \mathrm{~m} \mathrm{~s}^{-1}<\hat{v}_{\theta}^{b o}<18.5 \mathrm{~ms}^{-1}$. This blow off limit is most likely strongly dependent on the injector design, and may be lower in a different thermal environment, at a different operating point, or in other combustors. This point is briefly discussed in the theoretical analysis presented in [6]. 


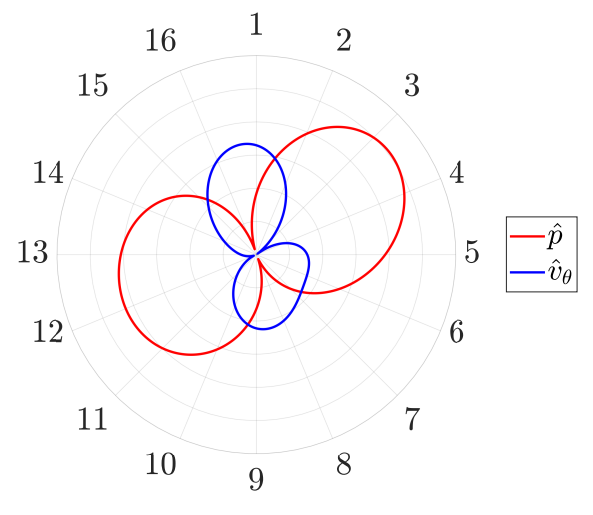

Fig. 10: Spatial structure of the acoustic pressure and azimuthal velocity fields at $t=106 \mathrm{~ms}$. The blue curve $\hat{v}_{\theta}$ corresponds to the azimuthal velocity field assuming a uniform temperature $T=1550 \mathrm{~K}$ in the combustion chamber. The velocity reconstruction method is detailed in Appendix A. The amplitude is shown by the radial position of the curve. For the velocity, each circle represents $5 \mathrm{~ms}^{-1}$, for the pressure, each circle represents 500 Pa.

\section{Modal Distortion Associated with Flame Blow Off}

It is now interesting to examine the pressure field as it evolves after the first flame blow off event. The acoustic pressure and velocity fields reconstructed in Fig. 9 and Fig. 10 indicate that there is an asymmetry between the two sides of the nodal line. Nonlinear low order modeling of combustion instabilities often relies on the modal expansion approach first introduced by Zinn and Powell [43], and explored by Culick $[44,45]$. In this framework, it is assumed that the spatial eigenmodes shapes are close to those of the acoustic eigenmodes of the system $\Psi_{n}(\boldsymbol{x})$. These modes are usually determined by assuming a low-Mach number flow with uniform mean pressure and no source or damping terms. The eignenmodes $\Psi_{n}(\boldsymbol{x})$ are then obtained as solutions to Eq. (7) [46] together with boundary conditions at the chamber walls, inlet plane and outlet.

$$
\nabla \cdot\left[\bar{c}^{2} \nabla \Psi_{n}(\boldsymbol{x})\right]+\omega_{n}^{2} \Psi_{n}(\boldsymbol{x})=0
$$

In the present study, combustion oscillations are coupled by a $1 \mathrm{~A} 1 \mathrm{~L}$ mode that is first order in the azimuthal direction and also first order in the axial direction. When all the flames are stabilized, the mean temperature in the annular chamber and the sound velocity are uniformly distributed. For a rigid backplane and open outlet the $1 \mathrm{~A} 1 \mathrm{~L}$ eigenmode is $\Psi_{1 A 1 L}(\theta, z)=(A \cos (\theta)+B \sin (\theta)) \cos (\pi z / 2 l)$ where $A, B$ are two real valued coefficients, $l$ is the length of the annular cavity, and $z$ the axial coordinate [46]. The spatial structure for the pressure field is that obtained in Fig. 9(i) for a relatively low level of oscillation. However, as the amplitude is increased and flames are blown off, the mean temperature distribution becomes non uniform and the modal structure is distorted.
In that situation the analytical solution of Eq. (7) leads to writing the eigenmode as a Fourier series with a fundamental and higher order coefficients. Appendix B indicates how they can be linked to the Fourier coefficients of the temperature field. This is one of the reasons why the higher order coefficients in the pressure expansion increase as the flames are blown off and indicates that this observation is a direct consequence of the inhomogeneity of the temperature distribution that arises from the blow off phenomenon.

In Appendix B, a short study is presented where the eigenfunction $\Psi$ and the eigenfrequency of acoustic modes in an open-closed annulus are computed. In this study, the azimuthal average of the sound velocity squared $\bar{c}^{2}$ is kept constant, but this field is deformed: $\bar{c}^{2}=c_{0}^{2}[1+2 \varepsilon \cos (\theta)]$. The eigenfunction $\Psi_{1 A 1 L}(\theta)$ is shown for different values of $\varepsilon$. It is distorted, and becomes asymmetric. Additionally, it is found that the eigenfrequencies monotonically decrease as the parameter $\varepsilon$ is increased. It is known from previous studies [47] that nonlinear features in the flame response can lead to shifts in the frequency of thermoacoustic oscillations. In distinction with standard modal expansion methods used in combustion instabilities analysis, it is found here that for large amplitudes of oscillations changes in the mean state of the system can distort the modal structures themselves, and shift the corresponding acoustic eigenfrequencies.

\section{PHASE OF THE FLAME RESPONSE TO THE PRES- SURE PERTURBATION}

It is known that the phase between the heat release rate in the flames and the local pressure plays plays a major role in combustion instability studies. When pressure and heat release rate are in phase energy is fed into the coupling mode. It is then interesting to determine the phase of the different flames with respect to the pressure. To this purpose one assumes that even in the spray combustion conditions that prevail in MICCA-Spray, most of the chemical conversion takes place in premixed flame elements and the light emission in the form of chemiluminescence is an approximate indicator of instantaneous heat release rate. Now, if the system were operating linearly one would find a uniform phase distribution which would remain uniform during the process. This is essentially the case at low amplitude levels, but the situation changes when the amplitude increases. The DMD images of Fig. 9 give an overall view of the phases for different amplitude levels. The evolution as the oscillation amplitude increases is clearly visible. It is also possible to obtain a direct quantitative time evolution of the phase from the different PM signals.

The phase reference in the DMD images of Fig. 9(f)9(h) is provided by microphone MC2, located in-between injectors 2 and 3 (see Fig. 1). In Fig. 9(f), flames 1 to 8 are green, indicating that the phase of the flame chemiluminescence to the acoustic pressure is approximately $-\pi / 4 \mathrm{rad}$ for these flames. Flames 9 to 16 are pink, in phase opposition to flames 1 to 8 , as as expected since the thermoacoustic oscillation features a standing azimuthal acoustic wave.

As the amplitude of the thermoacoustic oscillation in- 


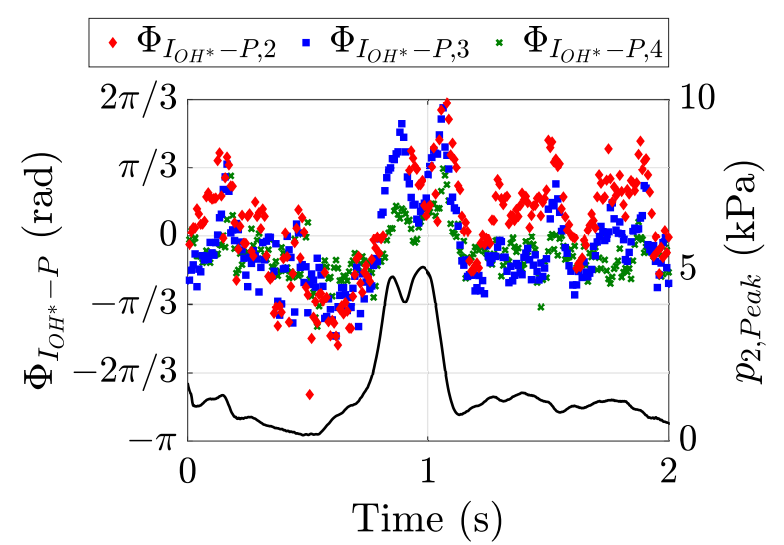

Fig. 11: Data for this figure are extracted from Fig. 4. The solid black line (right axis) is the instantaneous amplitude of the pressure at the location of injector 2 . The dots show the time resolved phase between the photomultiplier signal and the pressure for injectors 2 (red, diamonds), 3 (blue, squares) and 4 (green, crosses). Flame 2 is blown-off during $0.82 \mathrm{~s}<$ $t<0.92 \mathrm{~s}$, so that no phase data is available.

creases, some flames are blown-off in the combustor. In Fig. 9(g)-9(h), the phase of the flames on the right side of the combustor (flame 2-5) increases and becomes positive $(\Phi \approx \pi / 6)$. Flames on the opposite side of the combustor are still in phase opposition. One may however observe from the color in the DMD, that flames 2, 5, 10 and 15 appear to be out of phase with respect to their neighbors. For example, the phase of flame 2 is $0.48 \mathrm{rad} \approx \pi / 6 \mathrm{rad}$ in Fig. $9(\mathrm{~g})$, and $0.93 \mathrm{rad} \approx \pi / 3 \mathrm{rad}$ in Fig. $9(\mathrm{~h})$, while the phase of flame 3 is $0.38 \mathrm{rad} \approx \pi / 8 \mathrm{rad}$ in Fig. $9(\mathrm{~g})$, and $0.54 \mathrm{rad} \approx \pi / 6 \mathrm{rad}$ in Fig. 9(h).

This analysis of the DMD images is now complemented by examining the PM signals from sequence (a) of Fig. 5 are used. In Fig. 11, the phases of the PM signals with respect to the pressure are shown for injectors 2, 3 and 4. They are deduced from a time resolved correlation method with blocks $16 \mathrm{~ms}$ long. The reference pressure amplitude plotted in the same figure indicates the overall level of the thermoacoustic oscillation. Flame 2 is blown-off between $t=0.82 \mathrm{~s}$ and $0.92 \mathrm{~s}$.

As observed in the DMD images, the phase between the chemiluminescence signal and the pressure is slightly negative when the level of the oscillation is low, positive when it is very high. All injectors feature roughly the same phase between chemiluminescence and pressure for lower levels of oscillation (during the first $0.75 \mathrm{~s}$ and the last $0.2 \mathrm{~s}$ of the sequence). During very high amplitude oscillations, around $0.8-1.1 \mathrm{~s}$, injectors 2 and 3 have nearly the same phase, but it is higher than that of injector 4. Again, after $t=1.1 \mathrm{~s}$, the phase of injector 2 is often higher than that of injectors 3 and 4.

Similar observations can be made on other sequences featuring the same type of very high amplitude oscillations induced by a standing acoustic mode, using either the pho- tomultiplier array, or the high speed imaging system. At lower oscillation amplitudes, the phase between chemiluminescence and pressure is slightly negative, and it becomes positive at higher oscillation amplitudes. In all cases flames on the same side of the nodal line do not all feature the same phase when the amplitude of the oscillation becomes large. The phase distribution is clearly affected by the amplitude of oscillation, a nonlinear feature that can be modeled in the Flame Describing Function [47] framework. It is also likely that the change in phase results from the transverse velocity fluctuations and maybe perhaps from the presence of nearby extinguished flames.

\section{CONCLUSION}

This article describes experiments carried out on a laboratory scale annular combustor comprising multiple injectors fed with liquid fuel. This investigation is concerned with dynamical phenomena observed when the system features a coupling between combustion and azimuthal acoustic modes giving rise to thermoacoustic oscillations. Experiments reveal a broad variety of dynamical features. In particular, very high amplitude oscillations coupled by the standing first azimuthal mode $1 \mathrm{~A} 1 \mathrm{~L}$ of the annular system are described. The azimuthal mode coupling is intermittent and gives rise to intense bursts featuring the peak amplitude levels of $6 \mathrm{kPa}$ to $7 \mathrm{kPa}$. These pressure excursions are sufficiently high to induce an extinction of flames located near the pressure nodal line. This phenomenon already observed in our previous experiments is here analyzed by combining pressure oscillation measurements and images of the flames.

It is found in this case that the frequency of oscillation sweeps a range of values extending more than $10 \%$ around the nominal modal frequency (from $800 \mathrm{~Hz}$ to $620 \mathrm{~Hz}$ for a typical frequency of $730 \mathrm{~Hz}$ ). During these high amplitude oscillations, the spin ratio remains at zero, indicating that the coupling mode is standing with some slight displacements of the nodal line. A new result is that the acoustic pressure field is distorted as the amplitude increases. Using the signals delivered by the waveguide microphone array plugged on the chamber backplane, it is possible to expand the pressure up to third order in spatial components to account for this distortion which leads to a relatively asymmetric acoustic field.

Flame blow off is observed for peak acoustic pressure amplitudes above $2 \mathrm{kPa}$. During a large amplitude oscillation reaching $6.1 \mathrm{kPa}$, five of the sixteen flames of the combustor are blown off. An important result is that the blow off pattern is also not symmetric, and it appears strongly linked to asymmetries in the acoustic field. A method to reconstruct the acoustic velocity field is proposed, providing a first estimate of the critical threshold value. The phases of the heat release rates in the different flames with respect to the local pressure are also not uniform among all flames on each side of the nodal line. Flame blow off creates temperature inhomogeneities within the combustor, which are shown to be one of the contributing factors to the observed shift in frequency and to the deformation of the acoustic mode. At high amplitude of oscillations, it is important to consider distor- 
tions of the modal distributions induced by inhomogeneitites in the mean flow characteristics, notably those affecting the temperature and the sound velocity.

In summary, the key points of this paper are:

1. a mechanism of flame blow off induced by large amplitude oscillations in an annular combustor;

2. an evolution of the pressure distribution as flames are extinguished, shown using higher order spatial harmonics for reconstruction;

3. an estimation of the transverse velocity fluctuation that induces the initial flame blow off;

4. and a demonstration of the impact of perturbations in the mean sound velocity field on the acoustic eigenfunctions.

\section{Acknowledgements}

This work benefited from the support of SafranTech (contract NF5Z-5100), CNRS, CentraleSupelec, ParisSaclay University, and of project FASMIC ANR-16-CE220013 and TIMBER ANR14-CE23-0009-01 of the French National Research Agency (ANR).

\section{References}

[1] Lefebvre, A. H., and Ballal, D. R., 2010. Gas turbine combustion : alternative fuels and emissions. Taylor \& Francis.

[2] Poinsot, T., 2017. "Prediction and control of combustion instabilities in real engines". Proceedings of the Comb. Inst., 36(1), pp. 1-28.

[3] Candel, S., Durox, D., Schuller, T., Palies, P., Bourgouin, J.-F., and Moeck, J. P., 2012. "Progress and challenges in swirling flame dynamics”. CR Mécanique, 340, pp. 758-768.

[4] Candel, S., Durox, D., Schuller, T., Bourgouin, J.-F., and Moeck, J. P., 2014. "Dynamics of swirling flames". Annu. Rev. Fluid Mech., 46, pp. 147-73.

[5] Bourgouin, J.-F., Durox, D., Moeck, J. P., Schuller, T., and Candel, S., 2014. "Characterization and modeling of a spinning thermoacoustic instability in an annular combustor equipped with multiple matrix injectors". $J$. Eng. Gas Turb. Power, 137(2), p. 021503.

[6] Prieur, K., Durox, D., Schuller, T., and Candel, S., 2017. "Strong azimuthal combustion instabilities in a spray annular chamber with intermittent partial blowoff". J. Eng. Gas Turb. Power, 140(3), p. 031503.

[7] Worth, N. A., and Dawson, J. R., 2013. "Self-excited circumferential instabilities in a model annular gas turbine combustor: Global flame dynamics". Proceedings of the Comb. Inst., 34(2), pp. 3127-3134.

[8] Worth, N. A., Dawson, J. R., Sidey, J. A., and Mastorakos, E., 2017. "Azimuthally forced flames in an annular combustor". Proceedings of the Comb. Inst., 36(3), pp. 3783-3790.

[9] Noiray, N., and Schuermans, B., 2013. "On the dynamic nature of azimuthal thermoacoustic modes in an- nular gas turbine combustion chambers". Proc. $R$. Soc. Lond. A, 469(2151).

[10] Krebs, W., Flohr, P., Prade, B., and Hoffmann, S., 2002. "Thermoacoustic stability chart for high-intensity gas turbine combustion systems". Combust. Sci. Technol., 174(7), pp. 99-128.

[11] Bothien, M. R., Noiray, N., and Schuermans, B., 2013. "A novel damping device for broadband attenuation of low-frequency combustion pulsations in gas turbines". J. Eng. Gas Turb. Power, 136(4), p. 041504.

[12] Wolf, P., Staffelbach, G., Gicquel, L. Y., Müller, J.-D., and Poinsot, T., 2012. "Acoustic and Large Eddy Simulation studies of azimuthal modes in annular combustion chambers". Combust. Flame, 159(11), pp. 33983413.

[13] Wolf, P., Balakrishnan, R., Staffelbach, G., Gicquel, L. Y. M., and Poinsot, T., 2012. "Using LES to study reacting flows and instabilities in annular combustion chambers". Flow Turbul. Combust., 88(1-2), pp. 191206.

[14] Staffelbach, G., Gicquel, L. Y., Boudier, G., and Poinsot, T., 2009. "Large Eddy Simulation of self excited azimuthal modes in annular combustors". Proc. Combust. Inst., 32(2), pp. 2909-2916.

[15] Mazur, M., Nygård, H. T., Dawson, J. R., and Worth, N. A., 2019. "Characteristics of self-excited spinning azimuthal modes in an annular combustor with turbulent premixed bluff-body flames". Proc. Combust. Inst., 37(4), pp. 5129-5136.

[16] Bauerheim, M., Cazalens, M., and Poinsot, T., 2015. "A theoretical study of mean azimuthal flow and asymmetry effects on thermo-acoustic modes in annular combustors". Proc. Combust. Inst., 35(3), pp. 32193227.

[17] Bauerheim, M., Salas, P., Nicoud, F., and Poinsot, T., 2016. "Symmetry breaking of azimuthal thermoacoustic modes in annular cavities: a theoretical study". J. Fluid Mech., 760, pp. 431-465.

[18] Ghirardo, G., and Juniper, M. P., 2013. "Azimuthal instabilities in annular combustors: standing and spinning modes". Proc. R. Soc. Lond. A, 469(2157).

[19] Orchini, A., Mensah, G. A., and Moeck, J. P., 2018. "Effects of Nonlinear Modal Interactions on the Thermoacoustic Stability of Annular Combustors". J. Eng. Gas Turb. Power, 141(2), p. 021002.

[20] Moeck, J. P., Durox, D., Schuller, T., and Candel, S., 2019. "Nonlinear thermoacoustic mode synchronization in annular combustors". Proc. Combust. Inst., 37(4), pp. 5343-5350.

[21] Laera, D., Schuller, T., Prieur, K., Durox, D., Camporeale, S. M., and Candel, S., 2017. "Flame Describing Function analysis of spinning and standing modes in an annular combustor and comparison with experiments". Combust. Flame, 184, pp. 136-152.

[22] Laera, D., and Camporeale, S. M., 2017. "A weakly nonlinear approach based on a distributed flame describing function to study the combustion dynamics of a full-scale lean-premixed swirled burner". J. Eng. Gas 
Turb. Power, 139(9), p. 091501.

[23] Laera, D., Prieur, K., Durox, D., Schuller, T., Camporeale, S. M., and Candel, S., 2017. "Impact of heat release distribution on the spinning modes of an annular combustor with multiple matrix burners". J. Eng. Gas Turb. Power, 139(5), p. 051505.

[24] Mensah, G. A., Magri, L., Orchini, A., and Moeck, J. P., 2018. "Effects of Asymmetry on Thermoacoustic Modes in Annular Combustors: A Higher-Order Perturbation Study". J. Eng. Gas Turb. Power, 141(4), p. 041030.

[25] Juniper, M. P., and Sujith, R., 2018. "Sensitivity and Nonlinearity of Thermoacoustic Oscillations". Annual Rev. Fluid Mech., 50(1), pp. 661-689.

[26] Bauerheim, M., Staffelbach, G., Worth, N. A., Dawson, J. R., Gicquel, L. Y., and Poinsot, T., 2015. "Sensitivity of LES-based harmonic flame response model for turbulent swirled flames and impact on the stability of azimuthal modes". Proc. Comb. Inst., 35(3), pp. 33553363.

[27] Fanaca, D., Alemela, P. R., Hirsch, C., Sattelmayer, T., and Schuermans, B., 2009. "Comparison of the flow fields of a swirl stabilised premixed burner in an annular and a single burner combustion chamber". In Proceedings of ASME Turbo Expo 2009, no. GT200959884.

[28] De Rosa, A. J., Peluso, S. J., Quay, B. D., and Santavicca, D. A., 2015. "The effect of confinement on the structure and dynamic response of lean-premixed, swirl-stabilized flames". J. Eng. Gas Turb. Power, 138(6), p. 051505.

[29] Bonciolini, G., Ebi, D., Doll, U., Weilenmann, M., and Noiray, N., 2019. "Effect of wall thermal inertia upon transient thermoacoustic dynamics of a swirl-stabilized flame". Proc. Combust. Inst., 37(4), pp. 5351-5358.

[30] Durox, D., Moeck, J. P., Bourgouin, J.-F., Morenton, P., Viallon, M., Schuller, T., and Candel, S., 2013. "Flame dynamics of a variable swirl number system and instability control". Combust. Flame, 160(9), pp. 17291742.

[31] Bourgouin, J.-F., Moeck, J., Durox, D., Schuller, T., and Candel, S., 2013. "Sensitivity of swirling flows to small changes in the swirler geometry". CR Mécanique, 341(1-2), pp. 211-219.

[32] O'Connor, J., Acharya, V., and Lieuwen, T., 2015. "Transverse combustion instabilities: Acoustic, fluid mechanic, and flame processes". Prog. Energy Combust., 49, pp. 1-39.

[33] Bauerheim, M., Nicoud, F., and Poinsot, T., 2016. "Progress in analytical methods to predict and control azimuthal combustion instability modes in annular chambers". Phys. Fluids, 28, p. 021303.

[34] Baillot, F., and Lespinasse, F., 2014. "Response of a laminar premixed V-flame to a high-frequency transverse acoustic field”. Combust. Flame, 161(5), pp. 1247-1267.

[35] Lespinasse, F., Baillot, F., and Boushaki, T., 2013. "Responses of V-flames placed in an HF transverse acous- tic field from a velocity to pressure antinode". $\quad C R$ Mécanique, 341(1-2), pp. 110-120.

[36] Durox, D., Prieur, K., Schuller, T., and Candel, S., 2016. "Different flame patterns linked with swirling injector interactions in an annular combustor". J. Eng. Gas Turb. Power, 138(10), p. 101504.

[37] Prieur, K., Durox, D., Schuller, T., and Candel, S., 2016. "A hysteresis phenomenon leading to spinning or standing azimuthal instabilities in an annular combustor". Combust. Flame, 175, pp. 283-291.

[38] Beer, J. M., and Chigier, N. A., 1972. Combustion aerodynamics.

[39] Mirat, C., Durox, D., and Schuller, T., 2014. “Analysis of the spray and transfer function of swirling spray flames from a multi-jet steam assisted liquid fuel injector". In Proceedings of the ASME Turbo Expo 2014, no. GT2014-25111.

[40] Mirat, C., Durox, D., and Schuller, T., 2015. "Stability analysis of a swirl spray combustor based on flame describing function". Proc. Combust. Inst., 35(3), pp. 3291-3298.

[41] Bourgouin, J.-F., Durox, D., Moeck, J. P., Schuller, T., and Candel, S., 2015. "A new pattern of instability observed in an annular combustor: The slanted mode". Proc. Combust. Inst., 35(3), pp. 3237-3244.

[42] Schmid, P. J., 2010. "Dynamic mode decomposition of numerical and experimental data". J. Fluid Mech., 656, pp. 5-28.

[43] Zinn, B., and Powell, E., 1970. "Application of the Galerkin Method in the Solution of Combustion Instability Problems". In Proceedings of the Nineteenth International Astronautical Congress on Propulsion, ReEntry Physics 3, pp. 59-73.

[44] Culick, F. E. C., 1976. "Nonlinear behavior of acoustic waves in combustion chambers-I". Acta Astronautica, 3(9-10), pp. 715-734.

[45] Culick, F. E. C., 1988. "Combustion Instabilities in Liquid-Fueled Propulsion System - an Overview". In AGARD 72B Specialists' Meeting of the Propulsion and Energetics Panel 450.

[46] Poinsot, T., and Veynante, D., 2012. Theoretical and numerical combustion, third Edition.

[47] Noiray, N., Durox, D., Schuller, T., and Candel, S., 2008. "A unified framework for nonlinear combustion instability analysis based on the flame describing function”. J. Fluid Mech., 615, p. 139.

\section{Appendix A: Computing the Acoustic Azimuthal Velocity Field from Pressure Signals}

The acoustic velocity may be deduced from the linearized momentum balance for an annular configuration. Assuming small disturbances, neglecting viscous stresses and body forces and effects of the local mean velocity, one has [46] 


$$
\rho_{0}(\theta, t) \frac{\partial v_{\theta}}{\partial t}=-\frac{1}{R} \frac{\partial p^{\prime}}{\partial \theta}
$$

Equation (4) is linearised at the first order in time around the instant $t_{0}$ in Eq. (9). $\omega(t)=2 \pi f(t)$ is the instantaneous angular frequency, $\alpha_{m}(t)$ the instantaneous growth rate of the $\mathrm{m}^{\text {th }}$ component, and $\beta(t)$ the instantaneous change in angular frequency.

$$
\begin{array}{r}
p^{\prime}(\theta, t)=\sum_{m=-M}^{M} a_{m}\left(t_{0}\right) e^{-i \omega\left(t_{0}\right) t_{0}} \\
\exp \left\{i\left[m \theta-\omega\left(t_{0}\right) \varepsilon+\beta\left(t_{0}\right) t_{0} \varepsilon\right]+\alpha_{m}\left(t_{0}\right) \varepsilon+O\left(\varepsilon^{2}\right)\right\} \\
\varepsilon=t-t_{0} \\
\alpha_{m}\left(t_{0}\right)=\frac{1}{a_{m}\left(t_{0}\right)} \frac{d a_{m}}{d t}\left(t_{0}\right) \\
\beta\left(t_{0}\right)=\frac{d \omega}{d t}\left(t_{0}\right)
\end{array}
$$

Solving for Eq. (8), one obtains the first order approximation of the velocity field around $t_{0}$. Note that this expression is valid even if the density field is not uniform. One simply has to use the local value of the density field.

$$
\begin{array}{r}
v_{\theta}=-\frac{1}{R \rho_{0}} \sum_{m=-M}^{M} \frac{i m a_{m}(t)}{\alpha_{m}\left(t_{0}\right)+i \beta\left(t_{0}\right)-i \omega\left(t_{0}\right)} \\
\exp \{i(m \theta-\omega(t) t)\}+O\left(\left(t-t_{0}\right)^{2}\right)
\end{array}
$$

In most instances, the terms $\alpha_{m}$ and $\beta$ can be neglected compared to the angular frequency $\omega$. The velocity field then takes the simpler form

$$
\begin{array}{r}
v_{\theta} \simeq \frac{1}{R \rho_{0}} \sum_{m=-M}^{M} \frac{m a_{m}(t)}{\omega(t)} \\
\exp \{i(m \theta-\omega(t) t)\}
\end{array}
$$

This expression was used to determine the velocity field in Fig. 10.

\section{Appendix B: Acoustic Eigenmodes in an Annular Com- bustor with an Inhomogeneous Temperature Field}

In order to solve Eq. (7) in the case of an annulus with an inhomogeneous sound velocity field, we assume that the sound velocity field is uniform in the axial z-direction and that it can be written as a Fourier expansion of the angular coordinate $\theta$. It is actually more convenient to expand the square of this velocity in the form

$$
b_{0}=1, \bar{c}^{2}(\theta, z)=\bar{c}^{2}(\theta)=c_{0}^{2} \sum_{m=-\infty}^{+\infty} b_{m} e^{i m \theta}
$$

where $c_{0}$ is a mean sound velocity and $b_{m}$ designate the Fourier series expansion coefficients.

One may now consider pressure eigenfunctions that can be written as the product of a function depending on the axial coordinate $g(z)$ and a function depending on the azimuthal coordinate $f(\theta)$. This last function may be written in the form of a Fourier expansion so that

$$
\Psi_{n}(\theta, z)=f(\theta) g(z)=\left(\sum_{k=-\infty}^{+\infty} a_{k} e^{i k \theta}\right) g(z)
$$

The separation of variables in the wave equation requires that $g(z)$ satisfies the following equation :

$$
\frac{d^{2} g}{d z^{2}}=-k_{z}^{2} g
$$

where the wavenumber $k_{z}$ depends on the boundary conditions at the backplane and outlet of the combustor, which are here assumed to be azimuthally invariant. Inserting these different expressions into the wave equation and assuming that the number of coefficients in the Fourier expansions can be limited to a finite value $M$, one obtains after some calculations a set of equations linking the Fourier coefficients $a_{k}$ and $b_{m}$ which may be written as a matrix eigenvalue equation:

$$
\frac{c_{0}^{2}}{R^{2}}\left(\mathcal{D} \mathcal{B D}+k_{z}^{2} R^{2} \mathcal{B}\right) \mathcal{A}=\omega_{n}^{2} \mathcal{A}
$$

where $R$ is the mean radius of the annulus, $M$ is the finite dimension of the decomposition, $\mathcal{D}$ is the $2 M+1$ diagonal matrix with coefficients $\llbracket-M, 1-M, \ldots, 1,0,1, \ldots, M-1, M \rrbracket$, $\mathcal{B}$ is a Toeplitz matrix containing the Fourier coefficient $b_{m}$ of the sound velocity field, and $\mathcal{A}$ is a vector containing the Fourier coefficient $a_{k}$ of the eigenfunction $\Psi_{n}$. The shape of Eq. (15) arises from a discrete convolution product between the Fourier expansion of the sound velocity squared and the eigenfunction $\Psi_{n}$. Note that, for a perfect gas, $\bar{c}^{2}=\gamma r T$, with $\gamma$ the specific heat ratio, $r$ the specific gas constant, and $T$ the temperature, so that $\bar{c}^{2}$ is proportional to the temperature if one assumes that $\gamma$ and $r$ are constant.

Eq. (15) can easily be solved using an eigenvalue solver. For illustration purposes, it was solved numerically for $\bar{c}^{2}=$ $c_{0}^{2}(1+2 \varepsilon \cos (\theta)), c_{0}=800 \mathrm{~m} \mathrm{~s}^{-1}, 0<\varepsilon<0.3$ using Matlab. An open-closed configuration of length $l+\Delta l=850 \mathrm{~mm}$ is considered, leading to $k_{z}=\pi / 2(l+\Delta l)$ for the first longitudinal mode. The shape of the field of $\bar{c}^{2}$ is shown in Fig. 12. 


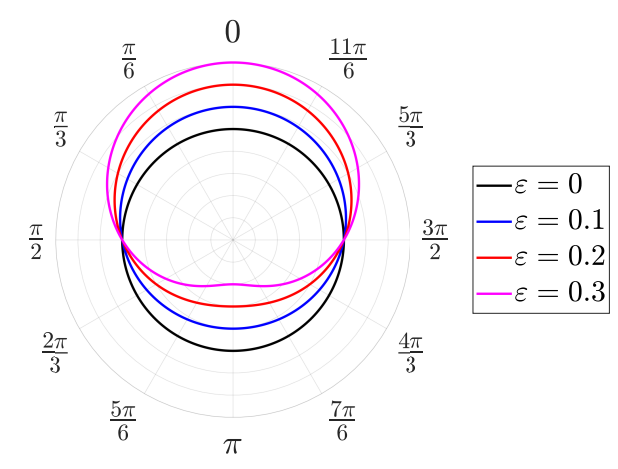

Fig. 12: Azimuthal distribution of $\bar{c}^{2}=c_{0}^{2}(1+2 \varepsilon \cos (\theta))$.

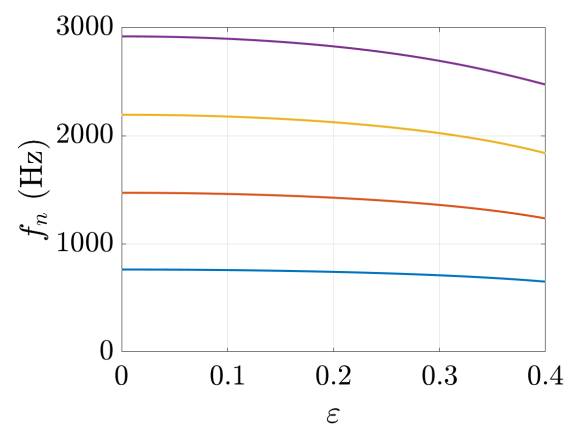

Fig. 13: First eigenfrequencies $\left(f_{1 A 1 L}\right.$ to $\left.f_{4 A 1 L}\right)$ solution of Eq. (15) for $0<\varepsilon<0.4$. The average value around the annulus of $\bar{c}^{2}$ is kept constant $\left(\bar{c}^{2}=c_{0}^{2}(1+2 \varepsilon \cos (\theta))\right)$.

These results were compared to the output of a 3D Helmholtz solver (COMSOL Multiphysics), and the relative difference on the computed eigenfrequency is $0.45 \%$ at most.

Figure 13 shows the evolution of the first four eigenfrequencies as a function of the parameter $\varepsilon$. The eigenfrequencies decrease monotonically with the increase of the inhomogeneities. The eigenfrequency of the $1 \mathrm{~A} 1 \mathrm{~L}$ mode varies from $f_{1 A 1 L}(\varepsilon=0)=765 \mathrm{~Hz}$ to $f_{1 A 1 L}(\varepsilon=0.3)=711 \mathrm{~Hz}$. That of the 4A1L mode from $f_{4 A 1 L}(\varepsilon=0)=2920 \mathrm{~Hz}$ to $f_{4 A 1 L}(\varepsilon=0.3)=2694 \mathrm{~Hz}$.

Figure 14 shows the shape of the pressure eigenfunction with various levels of sound velocity asymmetries $\varepsilon$. The pressure distribution becomes more asymmetric as $\varepsilon$, which defines the inhomogeneity in temperature, increases. The appearance of the pressure field is reminiscent of that plotted in Fig. 9, where one flame has been blown-off.

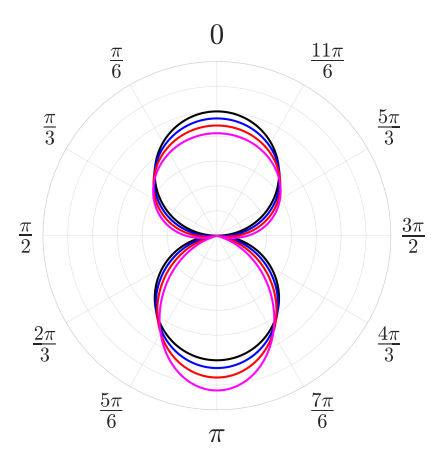

Fig. 14: Azimuthal pressure eigenfunction $\Psi_{n}$ solution of Eq. (15) for the fields of $\bar{c}^{2}$ shown in Fig. 12. 